\title{
III. Der Sonderfall zeitgleicher Reziprozität
}

Das sozialwissenschaftlich konzipierte Konstrukt der Reziprozität funktionierte am Hof - so die These - nach dem Prinzip der Ehre. Reziprokes Schenken am Hof dürfte sich also aller Voraussicht nach nur in der Minderzahl der Schenkvorgänge in der direkten Entsprechung von Gabe und Gegengabe niedergeschlagen haben ${ }^{1}$. Tatsächlich scheint die Überlieferung dies zu bestätigen, wie Tabelle 16 zeigt. Aufgefuihrt sind alle faßbaren Vorgänge der Zeit von 1381 bis 1422, die durch das zeitgleiche Aufeinandertreffen von Gabe und

\begin{tabular}{|c|c|c|c|}
\hline Jahr & Anzahl & Jahr & Anzahl \\
\hline 1381 & - & 1402 & 10 \\
\hline 1382 & - & 1403 & 11 \\
\hline 1383 & - & 1404 & 10 \\
\hline 1384 & 4 & 1405 & - \\
\hline 1385 & - & 1406 & - \\
\hline 1386 & 2 & 1407 & - \\
\hline 1387 & 2 & 1408 & - \\
\hline 1388 & 6 & 1409 & - \\
\hline 1389 & 9 & 1410 & - \\
\hline 1390 & 15 & 1411 & 2 \\
\hline 1391 & 7 & 1412 & 2 \\
\hline 1392 & 6 & 1413 & 2 \\
\hline 1393 & 5 & 1414 & 8 \\
\hline 1394 & 23 & 1415 & - \\
\hline 1395 & - & 1416 & - \\
\hline 1396 & 16 & 1417 & 2 \\
\hline 1397 & 5 & 1418 & - \\
\hline 1398 & 26 & 1419 & 9 \\
\hline 1399 & 2 & 1420 & - \\
\hline 1400 & 4 & 1421 & - \\
\hline 1401 & 12 & 1422 & - \\
\hline
\end{tabular}

Tab. 16: Zeitgleich-reziproke Schenkvorgänge 1381-1422

Gegengabe als direktes reziprokes Schenken definiert werden können, wobei sowohl Gabe als auch Gegengabe in die Auswertung eingegangen sind ${ }^{2}-$ einschränkend muß auch hier an die Überlieferungslücken erinnert werden.

Korrekterweise sollte freilich nur vom zeitgleichen Aufeinandertreffen zweier Gaben unter Beteiligung von nur zwei Personen gesprochen werden, denn es ist auf statistisch-deskriptiver Ebene im Unterschied zur statistischanalytischen Herangehensweise nicht feststellbar, ob im Einzelfall aus Gewohnheit, in Antizipation künftiger Geschenke oder in Reaktion auf ein Vor-

1 Siehe oben S. 124ff., insbes. S. 125, Anm. 20.

2 Nicht zuletzt auch deshalb, weil nicht zu bestimmen ist, was Gabe, was Gegengabe war. Ein Problem, das sich logischerweise bei fast allen zeitgleich-reziproken Vorgängen stellt. 
jahresgeschenk geschenkt worden ist. Es werden bei dem vorliegenden Untersuchungsschritt aber nicht die Schenkmotive zur Definition der einzelnen Schenkvorgänge zugrundegelegt, die einer statistisch-analytischen Betrachtung ja durchaus zugänglich sind, sondern die durch das zeitgleiche Aufeinandertreffen von Gabe und Gegengabe als reziprok definierten Schenkentscheidungen. Erst nach Feststellung dieser reziproken Schenkvorgänge soll in einem zweiten Schritt in Anlehnung an die oben gewonnenen Ergebnisse und mit Blick auf die Personen und Personenkreise, die die reziproken Schenkbeziehungen eingegangen sind, über Motive oder Motivstrukturen nachgedacht werden.

Ungerade Zahlenangaben in Tabelle 16 resultieren daraus, daß einige Beschenkte oder Schenker mehrere Geschenke derselben Person erhalten oder an dieselbe Person vergeben hatten. Insgesamt handelt es sich um 200 Schenkvorgänge ${ }^{3}$. Das sind bei den knapp $7.000(+x)$ dokumentierten Schenkvorgängen weniger als $3 \%$. Der Wert der reziproken Geschenke liegt allerdings bei $88.4641 .6 \mathrm{~d} .(+\mathrm{x})^{4}$, immerhin also etwa $17 \%$ des Gesamtvolumens von 518.5701 .12 s. $(+x)$. Dabei sind für fast die Hälfte der Geschenke keine Wertangaben zu machen; dennoch steht zu vermuten, daß der Prozentsatz tatsächlich nicht wesentlich höher sein wird, weil ja auch für die nicht reziproken Schenkvorgänge zu einem großen Teil keine Kosten notiert sind. Der hohe Gesamtwert scheint aber darauf hinzudeuten, daß hier besonders ausgeprägte Situationen der Konkurrenz um Anerkennungszuweisungen zugrundeliegen könnten.

Die Anzahl dieser Schenkvorgänge im Verbund mit den Jahresgesamtwerten zeigt zunächst Übereinstimmung mit dem weiter oben genannten Befund, der besagt, daß der eigentliche Höhepunkt des Geschenkverkehrs zwischen 1393 und 1405 lag mit einem kleinen Vorläufer in den Jahren von 1387

3 Die in Anhang - Katalog/Jahreslisten aufgeführten Positionen: 1384, Nr. 82-84 und 94; -1386, Nr. 167 und 181; - 1387, Nr. 204 und 237; - 1388, Nr. 240, 247, 254, 255, 274, 275; - 1389, Nr. 289, 294, 297f., 307f., 31 1, 314, 316; - 1390, Nr. 328-331, 334, 356, 361, 367f., 374f., 378f., 385f.; - 1391, Nr. 409, 427, 431, 439, 444, 460f.; - 1392, Nr. 490, 492, 502, 504, 508f.; - 1393, Nr. 548, 554, 566f., 575; - 1394, Nr. 605-607, 609-613, 615f, 620-623, 625, 627f., 633f., 642f., 646f.; - 1396, Nr. 699, 701-703, 713-715, 719, 721, 730, 733, 738f., 743f., 768; - 1397, Nr. 781f., 786, 803, 824; - 1398, Nr. 869, 871-873, 876f., 879, 881, 883f., 893, 897-899, 901, 911, 915, 924f., 935f., 995-997, 1000f.; - 1399, Nr. 1004 und 1006; - 1400, Nr. 1020, 1022, 1024, 1027; - 1401, Nr. 1064f., 1069, 1073, 1082f., 1095, 1098, 1106, 1113, 1116f; - 1402, Nr. 1153-1155, 1165, 1172-1174, 1186, 1192, 1209; 1403 , Nr. 1227, 1231f., 1236-1239, 1249, 1261, 1264f.; - 1404, Nr. 1281, 1286, 1289f., 1293, 1297, 1305, 1311-1313; - 1411, Nr. 1507, 1537; - 1412, Nr. 1544, 1549; - 1413, Nr. 1585,1600 ; - 1414, Nr. 1612, 1627f., 1633f., 1640, 1642f.; - 1417, Nr. 1699, 1701; 1419, Nr. 1716-1718, 1729f., 1732, 1735, 1745, 1755.

4 Einbezogen ist der Gesamtwert in Höhe von 3.600 1. des Geschenkes, das Karl VI. Philipp dem Kühnen 1397 machte, von dem der König aber nur 2.000 1. zahlte, Philipp den Rest trug, siehe Anhang - Katalog/Jahreslisten, Nr. 786. 
bis 13935 . Werden die Jahre 1384, 1386 und 1387 mit ihren relativ niedrigen Wertumfängen vernachlässigt, so lag der Beginn des reziproken Geschenkverkehrs am ersten Januar 1389, folgte also der Übernahme der Regierung durch Karl VI. 1388 (der übrigens auch erst 1389 mit einer nicht weiter bestimmbaren Gabe in diesen reziproken Geschenketausch eintrat ${ }^{6}$ ), wobei die höchsten Werte für die Jahre 1389, 1394, 1396, 1398 und 1404 genannt werden können. Auch der fast auf Dauer abrupte Abbruch des reziproken Geschenkaustausches nach dem Januar 1404 stimmt überein mit der oben gemachten Feststellung, daß dieses Jahr offensichtlich eine Zäsur im höfischen Geschenkverkehr gebildet hat und mit dem Tod Philipps des Kühnen zusammenhängt und zu erklären ist.

Tabelle 17 reiht Schenker und Beschenkte nach Häufigkeit ihrer Teilnahme an reziproken Schenkvorgängen von 1381 bis 1422 (die Ziffern in den Klammern nennen die Ränge, die die jeweils betreffenden Personen nach der Häufigkeit ihres Auftretens als Schenker und Beschenkte im Gesamtgeschenkverkehr einnahmen, wenn sie in reziproken Schenkbeziehungen häufiger als zweimal vertreten waren).

Es handelt sich um 37 namentlich bekannte Personen, die bis auf wenige Ausnahmen (die Brüder Limburg, die Brüder Rapondi und Gilles Malet, von denen oben schon die Rede war, sowie Pasqual de Paloiz, ein Genueser Händler) zur höchsten Hierarchieebene des Königreiches oder anderer Höfe zu zählen sind. Die Zahl derer, die nicht zum allerengsten Kreis an der Spitze des Königreiches zu zählen sind und nicht hochadlige Familienmitglieder waren, ist relativ gering - zumal hier noch keine Wertangaben eingegangen sind, sondern nur Bindungshäufigkeiten. »Familie« wurde bei dieser Vorgehensweise definiert nach Zugehörigkeit zu dem jeweiligen Haus, so daß zu den familiären Nahverhältnissen beispielsweise nicht die Beziehung Karl VI./Ludwig von Orléans gezählt worden ist. Auf den vorderen Plätzen rangieren dann auch bis auf Guy und Guillaume de La Trémoille, die einflußreichen Kammerherren Burgunds, oder Marie de Sully und Jeanne de Peschin, die Favoritinnen Philipps des Kühnen und Johanns Ohnefurcht, nur Familienangehörige (wiewohl auch die anderen genannten Personen selbstverständlich durch besondere Nahverhältnisse ausgezeichnet sind), denen unterstellt werden kann, daß ihr Ehrzuweisungspotential geringer ist als die Bindungskraft familiärer Solidarität ${ }^{7}$.

5 Siehe die Übersicht im vorliegenden Kap. Abschn. I.

6 Dieses Geschenk ist bekannt, weil eine burgundische Rechnung eine Belohnung der beiden Boten des Königs in Höhe von $150 \mathrm{fr}$. ausweist. Siehe Anhang - Katalog/Jahreslisten, Nr. 297.

7 Siehe auch den entspr. Befund oben S. $279 f$. 


\begin{tabular}{|c|c|c|c|c|c|}
\hline Rang & Teilnehmer & Zahl & Rang & Teilnehmer & Zahl \\
\hline$\overline{1}$ & Philipp der Kühne (1/3) & 113 & 15 & Charles d'Artois & 2 \\
\hline 2 & Ludwig von Orléans (3/4) & 52 & 15 & Clemens VII. & 2 \\
\hline 3 & Karl VI. $(5 / 2)$ & 35 & 15 & Dino Rapondi & 2 \\
\hline 4 & Johann Ohnefurcht $(2 / 7)$ & 32 & 15 & Gilles Malet & 2 \\
\hline 5 & Johann von Berry $(8 / 1)$ & 31 & 15 & Guy de La Rochefoucauld & 2 \\
\hline 6 & Isabeau de Bavière $(6 / 6)$ & 20 & 15 & Giacomo Rapondi & 2 \\
\hline 7 & Valentina Visconti (4/9) & 18 & 15 & Jean Canard & 2 \\
\hline 8 & $\begin{array}{l}\text { Margarete von Flandern } \\
(10 / 5)\end{array}$ & 16 & $\begin{array}{l}15 \\
15\end{array}$ & $\begin{array}{l}\text { Johann V. von Bretagne } \\
\text { Johann von Frankreich }\end{array}$ & $\begin{array}{l}2 \\
2\end{array}$ \\
\hline 9 & Guy de La Trémoille (13/11) & 11 & 15 & Karl I. von Bourbon & 2 \\
\hline 10 & Margarete von Bayern (12/8) & 7 & 15 & Karl III. von Navarra & 2 \\
\hline 11 & $\begin{array}{l}\text { Guillaume de La Trémoille } \\
(18 / 15)\end{array}$ & 6 & $\begin{array}{l}15 \\
15\end{array}$ & $\begin{array}{l}\text { Limburg, Gebr. } \\
\text { Louis de Vendôme }\end{array}$ & $\begin{array}{l}2 \\
2\end{array}$ \\
\hline 11 & $\begin{array}{l}\text { Ludwig II. von Bourbon } \\
(14 / 10)\end{array}$ & 6 & $\begin{array}{l}15 \\
15\end{array}$ & $\begin{array}{l}\text { Ludwig der Bärtige } \\
\text { Ludwig von Guyenne }\end{array}$ & $\begin{array}{l}2 \\
2\end{array}$ \\
\hline 12 & Marie de Sully (16/13) & 5 & 15 & Manuel II. Palaiologos & 2 \\
\hline 13 & Ludwig von Male (14/22) & 4 & 15 & Marie de Berry & 2 \\
\hline 14 & Jeanne de Peschin (20/25) & 3 & 15 & Pasqual de Paloiz & 2 \\
\hline 15 & Anton von Burgund & 2 & 15 & Pierre de La Trémoille & 2 \\
\hline 15 & Archambaut de Foix & 2 & 15 & Renier Pot & 2 \\
\hline
\end{tabular}

Tab. 17: Teilnehmer zeitgleich-reziproker Schenkvorgänge nach Häufigkeit

Es steht also zu vermuten, daß sich familiäre Bindungen als Schenkanreiz zumindest quantitativ in größerem Ausmaß in dieser Sonderform des Geschenkverkehrs niedergeschlagen haben könnten, so daß reziproke Anerkennungszuweisungsverhältnisse außerhalb dieser Bereiche zu suchen sein werden. Es ist also zu prüfen, ob sich der reziproke Austausch von Geschenken tatsächlich überwiegend in den einzelnen Familien abgespielt hat und damit den entsprechenden Nahverhältnissen zugewiesen werden kann oder ob auch konkurrierende Häuser wie Orléans und Burgund einen regelmäßigen gegenseitigen Geschenkverkehr gepflegt haben.

Weiteren Aufschluß gibt eine Beobachtung der Paarungen, die in Tabelle 18 aufgefuihrt sind, wobei Mehrfachbeschenkte mit entsprechenden Zahlenangaben versehen sind. Familiäre Nahverhältnisse stehen kursiv mit Ausnahme der Beziehungen zum König wegen seiner besonderen Position und wegen seiner außergewöhnlichen Situation.

Es ist zu sehen, daß von 91 jahrweise bestehenden reziproken Bindungen (nicht zu verwechseln mit der Gesamtzahl der reziproken Schenkbeziehungen) nur 16 den familiären Nahverhältnissen zuzurechnen sind, 18 - also nicht ganz $20 \%$-, wenn die Paarungen Philipp der Kühne/Marie de Sully und Johann Ohnefurcht/Jeanne de Peschin einbezogen werden. Damit scheint "Familie« offensichtlich nicht das Hauptschenkmotiv zu sein, ist aber, so darf angenommen werden, doch in einem Fünftel der Fälle von großer Bedeutung 


\begin{tabular}{|c|c|c|}
\hline Jahr & Schenkbeziehungen & \\
\hline 1384 & $\begin{array}{l}\text { Ludwig von Male } \\
\text { Ludwig von Male }\end{array}$ & $\begin{array}{l}\text { Margarete von Flandern } \\
\text { Philipp der Kühne }\end{array}$ \\
\hline 1386 & Philipp der Kühne & Marie de Sully \\
\hline 1387 & Philipp der Kühne & Pasqual de Paloiz \\
\hline 1388 & $\begin{array}{l}\text { Philipp der Kühne } \\
\text { Philipp der Kühne } \\
\end{array}$ & $\begin{array}{l}\text { Guy de La Trémoille } 2 \\
\text { Margarete von Bayern } 2\end{array}$ \\
\hline 1389 & $\begin{array}{l}\text { Philipp der Kühne } \\
\text { Philipp der Kühne } \\
\text { Philipp der Kühne } \\
\text { Philipp der Kühne }\end{array}$ & $\begin{array}{l}\text { Guy de La Trémoille } 2 \\
\text { Johann von Berry } \\
\text { Karl VI. } \\
\text { Ludwig II. von Bourbon }\end{array}$ \\
\hline 1390 & $\begin{array}{l}\text { Karl VI. } \\
\text { Philipp der Kühne } 2 \\
\text { Philipp der Kühne } \\
\text { Philipp der Kühne } \\
\text { Philipp der Kühne } \\
\end{array}$ & $\begin{array}{l}\text { Isabeau de Bavière } \\
\text { Guillaume de La Trémoille } 2 \\
\text { Johann von Berry } 2 \\
\text { Karl VI. } 2 \\
\text { Margarete von Flandern } 2 \\
\end{array}$ \\
\hline 1391 & $\begin{array}{l}\text { Karl VI. } \\
\text { Philipp der Kühne } \\
\text { Philipp der Kühne } \\
\text { Philipp der Kühne }\end{array}$ & $\begin{array}{l}\text { Isabeau de Bavière } \\
\text { Johann von Berry } \\
\text { Karl VI. } \\
\text { Margarete von Flandern }\end{array}$ \\
\hline 1392 & $\begin{array}{l}\text { Isabeau de Bavière } \\
\text { Johann Ohnefurcht } \\
\text { Karl VI. }\end{array}$ & $\begin{array}{l}\text { Ludwig (von Orléans) } \\
\text { Margarete von Bayern } \\
\text { Isabeau de Bavière } \\
\end{array}$ \\
\hline 1393 & \begin{tabular}{|l|} 
Philipp der Kühne \\
Philipp der Kühne \\
\end{tabular} & $\begin{array}{l}\text { Johann von Berry } 2 \\
\text { Ludwig von Orléans }\end{array}$ \\
\hline 1394 & $\begin{array}{l}\text { Clemens VII. } \\
\text { Johann Ohnefurcht } \\
\text { Philipp der Kühne } \\
\text { Philipp der Kühne } \\
\text { Philipp der Kühne } \\
\text { Philipp der Kühne } \\
\text { Philipp der Kühne } \\
\text { Philipp der Kühne } \\
\text { Philipp der Kühne } \\
\text { Philipp der Kühne } \\
\end{array}$ & $\begin{array}{l}\text { Philipp der Kühne } \\
\text { Margarete von Bayern } \\
\text { Guillaume de La Trémoille } \\
\text { Guy de La Trémoille } \\
\text { Jean Canard } \\
\text { Johann von Berry } \\
\text { Karl VI. } 2 \\
\text { Margarete von Flandern } 2 \\
\text { Pierre de La Trémoille } \\
\text { Renier Pot }\end{array}$ \\
\hline 1396 & $\begin{array}{l}\text { Ludwig von Orléans } \\
\text { Philipp der Kühne } \\
\text { Philipp der Kühne } \\
\text { Philipp der Kühne } \\
\text { Philipp der Kühne } \\
\text { Philipp der Kühne } \\
\text { Philipp der Kühne } \\
\text { Philipp der Kühne } \\
\end{array}$ & $\begin{array}{l}\text { Valentina Visconti } \\
\text { Gilles Malet } \\
\text { Isabeau de Bavière } \\
\text { Johann von Berry } \\
\text { Karl VI. } \\
\text { Ludwig von Orléans } 2 \\
\text { Ludwig von Orléans } \\
\text { Margarete von Flandern } 2 \\
\end{array}$ \\
\hline 1397 & $\begin{array}{l}\text { Guy de La Trémoille } \\
\text { Philipp der Kühne }\end{array}$ & $\begin{array}{l}\text { Marie de Sully } 2 \\
\text { Karl VI. }\end{array}$ \\
\hline 1398 & $\begin{array}{l}\text { Isabeau de Bavière } \\
\text { Johann Ohnefurcht } \\
\text { Karl VI. } \\
\text { Ludwig von Orléans } \\
\text { Ludwig von Orléans } \\
\text { Ludwig von Orléans } \\
\text { Philipp der Kühne } \\
\text { Philipp der Kühne }\end{array}$ & $\begin{array}{l}\text { Valentina Visconti } \\
\text { Ludwig von Orléans } \\
\text { Ludwig von Orléans } \\
\text { Isabeau de Bavière } \\
\text { Karl III. von Navarra } \\
\text { Ludwig II. von Bourbon } \\
\text { Guy de La Rochefoucauld } \\
\text { Isabeau de Bavière }\end{array}$ \\
\hline
\end{tabular}




\begin{tabular}{|c|c|c|}
\hline & $\begin{array}{l}\text { Philipp der Kühne } \\
\text { Philipp der Kühne } \\
\text { Valentina Visconti } \\
\text { Valentina Visconti } \\
\end{array}$ & $\begin{array}{l}\text { Ludwig II. von Bourbon } \\
\text { Ludwig von Orléans } \\
\text { Karl VI. } 2 \\
\text { Ludwig von Orléans } 2\end{array}$ \\
\hline 1399 & Johann Ohnefurcht & Ludwig von Orléans \\
\hline 1400 & $\begin{array}{l}\text { Johann Ohnefurcht } \\
\text { Philipp der Kühne }\end{array}$ & $\begin{array}{l}\text { Ludwig von Orléans } \\
\text { Dino Rapondi }\end{array}$ \\
\hline 1401 & $\begin{array}{l}\text { Isabeau de Bavière } \\
\text { Karl VI. } \\
\text { Ludwig von Orléans } \\
\text { Ludwig von Orléans } \\
\text { Ludwig von Orléans } \\
\text { Philipp der Kühne }\end{array}$ & $\begin{array}{l}\text { Valentina Visconti } \\
\text { Valentina Visconti } \\
\text { Johann von Berry } \\
\text { Johann Ohnefurcht } \\
\text { Valentina Visconti } \\
\text { Ludwig von Orléans }\end{array}$ \\
\hline 1402 & $\begin{array}{l}\text { Johann Ohnefurcht } \\
\text { Ludwig von Orléans } \\
\text { Ludwig von Orléans } \\
\text { Philipp der Kühne } \\
\text { Philipp der Kühne } \\
\end{array}$ & $\begin{array}{l}\text { Manuel II. Palaiologos } \\
\text { Johann Ohnefurcht } \\
\text { Valentina Visconti } \\
\text { Johann von Berry } \\
\text { Ludwig von Orléans }\end{array}$ \\
\hline 1403 & $\begin{array}{l}\text { Ludwig von Orléans } \\
\text { Ludwig von Orléans } \\
\text { Philipp der Kühne } \\
\text { Philipp der Kühne } \\
\text { Philipp der Kühne } \\
\end{array}$ & $\begin{array}{l}\text { Johann von Berry } \\
\text { Johann Ohnefurcht } \\
\text { Giacomo Rapondi } \\
\text { Ludwig von Orléans } \\
\text { Margarete von Flandern } 2 \\
\end{array}$ \\
\hline 1404 & $\begin{array}{l}\text { Johann Ohnefurcht } \\
\text { Philipp der Kühne } \\
\text { Philipp der Kühne } \\
\text { Philipp der Kühne }\end{array}$ & $\begin{array}{l}\text { Ludwig von Orléans } 2 \\
\text { Anton von Burgund } \\
\text { Johann von Berry } \\
\text { Karl VI. } 3 \\
\end{array}$ \\
\hline 1411 & Johann von Berry & Limburg, Gebrüder \\
\hline 1412 & \begin{tabular}{|l|} 
Karl VI. \\
\end{tabular} & Isabeau de Bavière \\
\hline 1413 & Johann Ohnefurcht & Ludwig von Guyenne \\
\hline 1414 & \begin{tabular}{|l|} 
Johann von Berry \\
Johann von Berry \\
Johann von Berry \\
Johann von Berry \\
\end{tabular} & $\begin{array}{l}\text { Karl I. von Bourbon } \\
\text { Charles d'Artois } \\
\text { Louis de Vendôme } \\
\text { Ludwig der Bärtige }\end{array}$ \\
\hline 1417 & Johann von Frankreich & Johann V. von Bretagne \\
\hline 1419 & $\begin{array}{l}\text { Johann Ohnefurcht } \\
\text { Johann Ohnefurcht } \\
\text { Johann Ohnefurcht } \\
\text { Johann Ohnefurcht }\end{array}$ & $\begin{array}{l}\text { Archambaut de Foix } \\
\text { Jeanne de Peschin } 2 \\
\text { Karl VI. } \\
\text { Marie de Berry }\end{array}$ \\
\hline
\end{tabular}

Tab. 18: Teilnehmer zeitgleich-reziproker Schenkbeziehungen nach Paarung

für reziproke Tauschbeziehungen gewesen ${ }^{8}$. In diesen Tauschbeziehungen schien aber, die oben auf statistisch-analytischem Weg erzielten Ergebnisse einbezogen, ein wichtiges Moment eine Rolle gespielt zu haben, daß selbst in diesen reziproken Beziehungen mit Gewohnheit im Schenken gegenüber Personen gleichen und höheren Ranges erklärt werden mag.

8 Damit wird wiederum das oben S. 279f. formulierte Ergebnis modifiziert, siehe aber auch oben S. 288. 
Werden die Paarungen, die durch Mehrfachschenkbeziehungen und mit Blick auf das jeweils einzelne Jahr als besonders intensiv definiert werden können, betrachtet, so ergibt sich das Bild der Tabelle 19. Es bestanden 18 dieser intensiven Schenkbeziehungen -, ebenfalls fast $20 \%$ der reziproken Tauschverhältnisse. Bis auf die Paarung Johann Ohnefurcht/Jeanne de Peschin endeten alle 1404 und nur an vier Tauschvorgängen war Philipp der Kühne

\begin{tabular}{|l|ll|}
\hline Jahr & Schenkbeziehungen & \\
\hline 1388 & $\begin{array}{l}\text { Philipp der Kühne } \\
\text { Philipp der Kühne }\end{array}$ & $\begin{array}{l}\text { Guy de La Trémoille 2 } \\
\text { Margarete von Bayern 2 }\end{array}$ \\
\hline 1389 & Philipp der Kühne & Guy de La Trémoille 2 \\
\hline 1390 & $\begin{array}{l}\text { Philipp der Kühne 2 } \\
\text { Philipp der Kühne } \\
\text { Philipp der Kühne } \\
\text { Philipp der Kühne }\end{array}$ & $\begin{array}{l}\text { Guillaume de La Trémoille 2 } \\
\text { Johann von Berry 2 } \\
\text { Karl VI. 2 } \\
\text { Margarete von Flandern 2 }\end{array}$ \\
\hline 1393 & Philipp der Kühne & Johann von Berry 2 \\
\hline 1394 & Philipp der Kühne & Margarete von Flandern 2 \\
\hline 1396 & $\begin{array}{l}\text { Philipp der Kühne } \\
\text { Philipp der Kühne }\end{array}$ & $\begin{array}{l}\text { Ludwig von Orléans 2 } \\
\text { Margarete von Flandern 2 }\end{array}$ \\
\hline 1397 & Guy de La Trémoille & Marie de Sully 2 \\
\hline 1398 & $\begin{array}{l}\text { Valentina Visconti } \\
\text { Valentina Visconti }\end{array}$ & Karl VI. 2 \\
Ludwig von Orléans 2
\end{tabular}

Tab. 19: Teilnehmer zeitgleich-reziproker Schenkvorgänge nach Intensität

nicht beteiligt. Der Burgunderherzog trat allerdings nach 1396 nur noch zweimal in Erscheinung und es läßt sich nur in der Überlieferungssituation ein plausibler Grund für diese Unterbrechung finden, wird beachtet, daß Philipp ansonsten sehr wohl auch weiterhin an reziproken Tauschvorgängen beteiligt war. Unter die Rubrik »Familie « fallen sieben Paarungen, das sind etwa $41 \%$. Damit ist prozentual gesehen der Anteil familiärer Schenkbeziehungen an intensiv gestalteten Schenkakten mehr als doppelt so hoch wie der Anteil familiärer Austauschbeziehungen an allen reziproken Schenkvorgängen. Nur handelte es sich in der Regel um die Paarung Philipp der Kühne/Margarete von Flandern und könnte als Ergebnis einer besonderen persönlichen Nahbeziehung ohne weiteren Erklärungsbedarf verbucht werden wie auch der $\mathrm{Ge}$ schenkaustausch mit seiner Schwiegertochter Margarete von Bayern 1388 (wiewohl die Bindung selbst natürlich Ergebnis politischer Überlegungen war) und die Beziehungen Guy de La Trémoille/Marie de Sully sowie Valentina Visconti/Ludwig von Orléans 1398 sowie der Austausch 1384 zwischen Philipp dem Kühnen, Margarete von Flandern und Ludwig von Male, zusätzlich allerdings auch 1419 derjenige zwischen Johann Ohnefurcht und Jeanne de Peschin. 
Bei den übrigen Tauschvorgängen ist zunächst die Beziehung Philipp der Kühne/Karl VI. auffällig. Der König erhielt 1404 von dem Burgunderherzog gleich zwei wertvolle Geschenke, eine Kollane und einen Balasrubin de Venise, die zusammen 3.700 l. gekostet hatten ${ }^{9}$, der König gab ihm 4.000 écus $^{10}$. Dies war eine äußerst ausgeglichene Reziprozität, die eher auf rational-ökonomische Überlegungen seitens des Burgunders schließen läßt als auf ein ausgeprägtes Streben nach Ehrzuweisungen. Andererseits war Philipp der erste Pair Frankreichs und der geringe Wertunterschied könnte auch ein Indiz für zwei nur noch graduell unterschiedliche Hierarchieebenen sein. Das Tauschverhalten des Jahres 1397 zwischen Philipp und Karl weist deutliche Parallelen auf. Philipp hatte dem König une ymaige de Saint Michel im Wert von $1.6501 .{ }^{11}$ geschenkt und er hatte 2.0001 . erhalten, so daß er sich - anders läßt sich dieser Umstand nicht ausdrücken - den Wunsch erfüllen konnte, eine 3.600 1. teure Annonciation de Nostre Dame d'or zu erwerben, die ihn dann nur noch $1.650 \mathrm{l}$. gekostet hat 12 .

In den Jahren 1390 und 1393 konnte Johann von Berry von seinem Bruder Philipp je zwei Geschenke entgegennehmen und 1396 sein Neffe, der Herzog von Orléans, ebenfalls. Während von den Gegengeschenken nur bekannt ist, $\mathrm{da} ß$ es sie gegeben hat, weil Philipp die Boten belohnt hatte ${ }^{13}$, hat der Burgunderherzog Berry 1390 einen grant tablel zu 1.5001 .14 und 1393 für insgesamt 1.2121 . einen saint Louis d'or ${ }^{15}$ sowie einen fermail d'or ${ }^{16}$ zukommen lassen - ausgesprochen höfische Gaben. Ludwig hatte 1396 une ymaige de saint Jehan und un saint Jean-Baptiste sur tablette de vermeil erhalten, die

9 Siehe Anhang - Katalog/Jahreslisten, Nr. 1311 und 1312.

10 Ebd., Nr. 1313 ist entspr. zu korrigieren. POCQUET DU HAUT-JUSSÉ, Les dons extraordinaires, 1939, S. 117 äußert schlicht die Annahme, Philipp habe seit einiger Zeit eine gewisse Vorliebe für Geldgeschenke gehabt, siehe hierzu Anhang - Katalog/Jahreslisten die nicht eindeutig zu datierende Nr. 1157. Philipp hatte sich im Dezember 1401 oder 1402 10.000 fr. aus der königlichen Kasse genommen "pour récompense de ses services que pour ses étrennes du jour de l'an précédent«, siehe POCQUET DU HAUT-JUSSÉ, Les dons extxraordinaires, 1939, S. 117, in der Datierung uneinig mit sich selbst: siehe POCQUET DU HAUT-JUSSÉ 1940, S. 108, Nr. 56. VAUGHAN 1962, S. 230 schließlich meint, won 12 December 1402 , he helped himself to 10,000 francs from the royal treasury in compensation for the fact that, on 1 January $1401[\ldots]$...

11 Anhang - Katalog/Jahreslisten, Nr. 824.

12 Ebd., Nr. 786.

13 Zu den Geschenken Berrys an Philipp 1390 ebd., Nr. 331 (überbracht von Perrinet, einem Kammerdiener Berrys, der von Philipp für seinen Dienst immerhin über $100 \mathrm{fr}$. bekam) und 1393 ebd., Nr. 548 (ein saint Louis d'or), zu dem Geschenk Ludwigs 1396 ebd., Nr. 713 (überbracht von Salmon, dem Barbier des Herzogs von Orléans, der 40 fl. erhielt).

14 Ebd., Nr. 374, möglicherweise identisch mit der nach COMMEAUX 1979, S. $245 f$. notierten Nr. 375.

15 Da Berry Philipp im selben Jahr ein gleiches Geschenk machte, drängt sich die Frage nach einer Verwechslung auf, aber die Belegstellen sind unterschiedlich.

16 Anhang - Katalog/Jahreslisten, Nr. 566. 
summiert mit $1.760 \mathrm{fl}$. in den burgundischen Rechnungen stehen 17 . Es ist anzunehmen, daß die gegenläufigen Geschenke der Herzöge in Wert und Aufmachung nicht sonderlich verschieden waren von denjenigen, die sie selbst erhalten hatten, so daß sich auf dieser Ebene über eine ausbalancierte Geschenkbilanz spekulieren ließe.

$\mathrm{Zu}$ beachten ist schließlich die Paarung Valentina Visconti/Karl VI. 1398. Das Geschenk des Königs ist wiederum nur durch die Verbuchung einer Botenentlohnung bekannt ${ }^{18}$. Valentina selbst hat Karl, zu dem sie bis zu ihrer Verbannung vom Hof im April 1393 ein bekannt gutes Verhältnis hatte, Geschenke für 1.9501 . überbringen lassen ${ }^{19}$. Es soll nicht unerwähnt bleiben, daß Valentina auch der Königin ein wertvolles Geschenk gemacht hatte, das mit 1.0241 . verrechnet worden ist ${ }^{20}$ und daß sie umgekehrt auch von Isabeau bedacht worden ist ${ }^{21}$.

Die auffälligsten aller Paarungen intensiver reziproker Schenkvorgänge sind jedoch diejenigen zwischen Philipp dem Kühnen und Guy und Guillaume de La Trémoille. Die Brüder de La Trémoille gehörten zu den vornehmsten burgundischen Rittern. Beide waren Kammerherren und Räte des Königs und des Burgunderherzogs, Guy premier chambellan und grand chambellan héréditaire Philipps des Kühnen, und beide, weit gereist und kampferprobt, ließen ihr Leben nach der Schlacht von Nikopolis, Guillaume in Gefangenschaft, Guy ereilte der Tod während der Rückreise auf Rhodos ${ }^{22}$. Der vierfach belegte Schenkvorgang von 1390 zwischen dem Herzog Burgunds und Guillaume de La Trémoille ist in diesem Sinn die stärkste aller intensiven reziprok-zeitgleichen Schenkbeziehungen. Guillaume schenkte Philipp zwei Pferde, die er durch Boten überbringen ließ, ung roncin und une haquenée, ein Schlachtroß und einen Zelter ${ }^{23}$, und empfing zwei Spangen, freilich zu dem relativ bescheidenen Wert von 600 1., obwohl er, wie gezeigt wurde, Aufnahme in den »inner circle« des höfischen Geschenkverkehrs gefunden hatte. Der Wert der Geschenke Philipps an Guy de La Trémoille 1388 ist mit 7201. auch nicht wesentlich höher gewesen, das Geschenk Guys nur durch den Bo-

Anhang - Katalog/Jahreslisten, Nr. 738 (1.400 fl.) und 739 (360 fl.).

Ebd., Nr. 881.

Ebd., Nr. 996, 997.

Ebd., Nr. 995.

Ebd., Nr. 873.

22 Siehe zu dem Geschlecht der Trémoille ConTAMINE, Philippe: Art. »La Trémoille«, in: LexMA, Bd. 5, 1991, Sp. 1748-1750 mit weiteren Nachweisen.

23 Anhang - Katalog/Jahreslisten, Nr. 328 und 329 (zwei Pferde sind als zwei Schenkvorgänge angesehen worden, natürlich ließe sich über eine solche Aufteilung streiten). Da Guillaume 1389/1390 in Preußen war (Beleg siehe oben S. 183, Anm. 346), kann es sich hier um einen noch höher zu bewertenden Schenkakt handeln, setzt er doch eine längerfristige Planung voraus. 
tenlohn überliefert ${ }^{24}$. 1389 hatte Philipp von seinem ersten Kammerherrn ebenfalls ein Pferd erhalten ${ }^{25}$, dieser hatte nun allerdings eine "fürstliche« Gabe bekommen, nämlich eine Spange und Tafelsilber im Gesamtwert von $1.4001 .^{26}$. Diese intensive Beziehung des Fürsten zu seinen engsten Beratern bestätigt die oben angeführte These Paravicinis, daß nicht auf verwandtschaftlichen Bindungen beruhende Treueverhältnisse stabiler scheinen ${ }^{27}$, in qualitativer Hinsicht.

Tabelle 20 gibt eine Aufstellung der Paarungen nach Häufigkeit und Wertzuweisungen nach Ausschluß aller Beziehungen ohne Wertangaben, sortiert nach absteigender Häufigkeit mit Werten $>1$. Es zeigt sich das in dieser Deutlichkeit überraschende Ergebnis, daß ausgerechnet die Paarung Johann Ohne-

\begin{tabular}{|l|l|l|}
\hline 8 & Johann Ohnefurcht & Ludwig von Orléans \\
\hline 7,5 & Johann von Berry & Philipp der Kühne \\
7,5 & Ludwig von Orléans & Philipp der Kühne \\
\hline 6,5 & Margarete von Flandern & Philipp der Kühne \\
\hline 4,5 & Philipp der Kühne & Karl VI. \\
4,5 & Guillaume de La Trémoille & Philipp der Kühne \\
4,5 & Ludwig von Orléans & Valentina Visconti \\
\hline 3 & Karl VI. & Philipp der Kühne \\
2,5 & Philipp der Kühne & Johann von Berry \\
2,5 & Karl VI. & Valentina Visconti \\
\hline 2 & Karl VI. & Isabeau de Bavière \\
2 & Margarete von Bayern & Johann Ohnefurcht \\
2 & Isabeau de Bavière & Karl VI. \\
2 & Johann von Berry & Ludwig von Orléans \\
2 & Isabeau de Bavière & Philipp der Kühne \\
2 & Isabeau de Bavière & Valentina Visconti \\
\hline 1,5 & Philipp der Kühne & Guy de La Trémoille \\
\hline 1,5 & Jeanne de Peschin & Johann Ohnefurcht \\
1,5 & Guy de La Trémoille & Marie de Sully \\
1,5 & Margarete von Bayern & Philipp der Kühne \\
\hline
\end{tabular}

Tab. 20: Zeitgleich-reziprok bestimmte Schenkbeziehungen nach Häufigkeit > 1

furcht/Ludwig von Orléans die häufigste aller reziprok bestimmten Schenkbeziehungen war, die von 1399 bis 1404 jedes Jahr praktiziert wurde, 1404 dann allerdings auch das letzte Mal. Handelte es sich hier um die Konkurrenz der beiden Häuser auf dem "Schenkfeld" als Appell an die Dritten oder um höfisch sublimierte Gegnerschaft?28 Trotz burgundisch-orléanistischer Spannungen ist der Geschenkverkehr zwischen Johann Ohnefurcht und Ludwig 
von Orléans bis 1404 nicht unterbrochen worden ${ }^{29}$ und obwohl für den Gabentausch zum neuen Jahr $1404^{30}$ keine Werte angegeben werden können, könnte diese Tatsache einerseits für die Vermutung sprechen, daß sich politische Kontroversen über einen bestimmten Punkt hinaus nicht in den höfischen Geschenkverkehr hinein verlängert haben ${ }^{31}$, andererseits im speziellen Fall für die politische Klugheit Philipps des Kühnen, schließlich aber auch dafür, daß sich das Motiv der Gewohnheit auch im Rahmen reziproker Schenkbeziehungen eher im Schenken gegenüber Personen gleichen und höheren Ranges manifestiert habe ${ }^{32}$. Eine weitere Interpretationsmöglichkeit wäre allerdings der Umstand, daß die sofortige Erwiderung unmittelbar entlastende Funktion hat ${ }^{33}$. Ludwig von Orléans und Johann Ohnefurcht hätten dann die der Interaktionsform des Schenkens immanente Intention, sich verpflichten zu lassen oder sich verpflichtet zu fühlen, erfolgreich negiert. Alle anderen Schenkbeziehungen bewegen sich im Rahmen der bislang erarbeiteten Ergebnisse nach Gruppenzugehörigkeit und Rangfolge und erneut wird die besondere Position der La Trémoilles erkennbar.

Abschließend sollen die Wertzuweisungen nach der Ordnung in Tabelle 21 betrachtet werden. Die mit einem + gekennzeichneten Paarungen sind einseitig gerichtete Schenkbeziehungen, die aus dem reziproken Zusammenhang herausgelöst wurden, weil für die Gegenseite keine Wertangaben vorlagen.

\begin{tabular}{|l|ll|l|}
\hline Jahr & Paarung & & Wert \\
\hline $1404+$ & Philipp der Kühne & Karl VI. & $7.700 \mathrm{fr}$. \\
1397 & Karl VI. & Philipp der Kühne & $5.250 \mathrm{l}$. \\
$1396+$ & Philipp der Kühne & Margarete von Flandern & $3.500 \mathrm{fl}$. \\
$1403+$ & Philipp der Kühne & Margarete von Flandern & $3.220 \mathrm{fr}$. \\
$1402+$ & Philipp der Kühne & Karl VI. & $3.000 \mathrm{fr}$. \\
$1394+$ & Philipp der Kühne & Margarete von Flandern & $2.700 \mathrm{fr}$. \\
$1403+$ & Ludwig von Orléans & Philipp der Kühne & 2.6501. \\
$1401-$ & Ludwig von Orléans & Philipp der Kühne & $2.600 \mathrm{fr}$. \\
1402 & Ludwig von Orléans & Valentina Visconti & $2.090 \mathrm{fr}$. \\
$1394+$ & Philipp der Kühne & Clemens VII. & $2.000 \mathrm{fr}$. d'or \\
$1398+$ & Valentina Visconti & Karl VI. & $1.950 \mathrm{fr}$. \\
$1404+$ & Anton von Burgund & Philipp der Kühne & $1.800 \mathrm{fr}$. \\
$1390+$ & Philipp der Kühne & Margarete von Flandern & $1.767 \mathrm{fr}$. \\
$13981-$ & Valentina Visconti & Ludwig von Orléans & $1.707 \mathrm{fr} .3 \mathrm{~d}$. \\
$1396+$ & Philipp der Kühne & Karl VI. & $1.700 \mathrm{fl}$. \\
$1396+$ & Philipp der Kühne & Ludwig von Orléans & $1.700 \mathrm{fr}$. \\
$1396+$ & Philipp der Kühne & Johann von Berry & $1.600 \mathrm{fl}$. \\
$1398+$ & Ludwig von Orléans & Karl VI. & $1.600 \mathrm{fr}$.
\end{tabular}

29 Vgl. auch ChAMPOLLION-FigÉAC 1844, S. 99 und MiROT 1938, S. 156 mit Anm. 6.

30 Anhang - Katalog/Jahreslisten, Nr. 1289, 1290, 1293.

31 Vgl. auch das bereits oben erwähnte Beispiel des Geschenkaustausches zwischen Ludwig XI. und Karl VII. nach KENDALL 1971, 74. AUTRAND, Jean de Berry, 2000, S. 484: »Le sourire, on le sait, est la politesse des princes«.

32 Siehe oben S. 279.

33 Siehe oben S. 125 mit Anm. 20. 


\begin{tabular}{|c|c|c|c|}
\hline $1390+$ & Isabeau de Bavière & Karl VI. & $1.534 \mathrm{fr}$. \\
\hline 1391 & Karl VI. & Philipp der Kühne & $1.512 \mathrm{fr}$. \\
\hline $1390+$ & Philipp der Kühne & Johann von Berry & $1.500 \mathrm{fr}$. \\
\hline $1404+$ & Philipp der Kühne & Johann von Berry & $1.500 \mathrm{fr}$ \\
\hline 1396 & Ludwig von Orléans & Valentina Visconti & 1.472 fr. d'or \\
\hline $1389+$ & Philipp der Kühne & Guy de La Trémoille & $1.400 \mathrm{fr}$. \\
\hline $1398+$ & Philipp der Kühne & Ludwig von Orléans & $1.400 \mathrm{fr}$. \\
\hline $1402+$ & Philipp der Kühne & Johann von Berry & $1.350 \mathrm{fr}$. \\
\hline $1402+$ & Philipp der Kühne & Ludwig von Orléans & $1.350 \mathrm{fr}$. \\
\hline $1401+$ & Ludwig von Orléans & Johann von Berry & $1.250 \mathrm{fr}$. \\
\hline $1393+$ & Philipp der Kühne & Johann von Berry & $1.212 \mathrm{fr}$. \\
\hline $1403+$ & Ludwig von Orléans & Johann von Berry & 1.2001 \\
\hline $1392+$ & Isabeau de Bavière & Karl VI. & $1.094 \mathrm{fr}$. \\
\hline $1394+$ & Philipp der Kühne & Karl VI. & 1.050 fr. d'or \\
\hline $1398+$ & Valentina Visconti & Isabeau de Bavière & $1.024 \mathrm{fr}$ \\
\hline $1394+$ & Philipp der Kühne & Johann von Berry & 1.0201 \\
\hline $1391+$ & Philipp der Kühne & Margarete von Flandern & $1.000 \mathrm{fr}$. \\
\hline 1391 & Isabeau de Bavière & Karl VI. & $930 \mathrm{fr}$. \\
\hline 1401 & Ludwig von Orléans & Valentina Visconti & 874 fr. \\
\hline $1389+$ & Philipp der Kühne & Karl VI. & 784 fr. 66 s. 3 d.t. \\
\hline 1394 & Johann Ohnefurcht & Margarete von Bayern & $790 \mathrm{fr}$. \\
\hline $1388+$ & Philipp der Kühne & Margarete von Bayern & $760 \mathrm{fr}$. \\
\hline $1388+$ & Philipp der Kühne & Guy de La Trémoille & $700 \mathrm{fr}$. \\
\hline $1390+$ & Philipp der Kühne & Karl VI. & $600 \mathrm{fr}$. \\
\hline $1413+$ & Ludwig von Guyenne & Johann Ohnefurcht & $550 \mathrm{fr}$. \\
\hline $1398+$ & Philipp der Kühne & Isabeau de Bavière & $522 \mathrm{fr}$. \\
\hline $1390+$ & Philipp der Kühne & Guillaume de La Trémoille & $500 \mathrm{fr}$. \\
\hline $1393+$ & Philipp der Kühne & Ludwig von Orléans & $500 \mathrm{fr}$. \\
\hline $1398+$ & Ludwig von Orléans & Isabeau de Bavière & 500 l.t. \\
\hline $1400+$ & Philipp der Kühne & Dino Rapondi & 500 fr. d'or \\
\hline $1419+$ & Karl VI. & Johann Ohnefurcht & 500 l.t. \\
\hline $1396+$ & Philipp der Kühne & Isabeau de Bavière & $480 \mathrm{fl}$. \\
\hline $1398+$ & Ludwig von Orléans & Ludwig II. von Bourbon & 453 fr. 6 s. 1 d. \\
\hline $1387+$ & Philipp der Kühne & Pasqual de Paloiz & $400 \mathrm{fr}$. \\
\hline $1398+$ & Philipp der Kühne & Ludwig II. von Bourbon & $378 \mathrm{fr}$. \\
\hline $1394+$ & Philipp der Kühne & Guillaume de La Trémoille & $350 \mathrm{fr}$. \\
\hline 1392 & Johann Ohnefurcht & Margarete von Bayern & 3301. \\
\hline $1398+$ & Ludwig von Orléans & Karl III. von Navarra & 310 fr. 8 s. 2 d. \\
\hline $1394+$ & Philipp der Kühne & Jean Canard & 292 fr. $10 \mathrm{~s}$. \\
\hline $1403+$ & Philipp der Kühne & Giacomo Rapondi & 300 l. d'or \\
\hline $1389+$ & Philipp der Kühne & Ludwig II. von Bourbon & 266 fr. 5 s.t. \\
\hline $1384+$ & Margarete von Flandern & Ludwig von Male & 2501. \\
\hline 1414 & Johann von Berry & Ludwig der Bärtige & 2231.5 s. 8 d.t. \\
\hline 1388 & Margarete von Bayern & Philipp der Kühne & $226 \mathrm{fr}$. \\
\hline $1384+$ & Philipp der Kühne & Ludwig von Male & 2251. \\
\hline $1396+$ & Philipp der Kühne & Gilles Malet & $200 \mathrm{fl}$. \\
\hline $1419+$ & Johann Ohnefurcht & Jeanne de Peschin & $200 \mathrm{fr}$. \\
\hline 1397 & Marie de Sully & Guy de La Trémoille & 175 fr. 10 s.t. \\
\hline $1414+$ & Johann von Berry & Charles d'Artois & 1581.2 s. 6 d.t. \\
\hline $1386+$ & Philipp der Kühne & Marie de Sully & 1601. \\
\hline $1414+$ & Johann von Berry & Louis de Vendôme & 144 l. 7 s. 6 d.t. \\
\hline $1401+$ & Valentina Visconti & Isabeau de Bavière & $150 \mathrm{fr}$. \\
\hline
\end{tabular}




\begin{tabular}{|c|c|c|c|}
\hline $\begin{array}{l}1401+ \\
1401+ \\
1414+ \\
1398+ \\
1411+\end{array}$ & $\begin{array}{l}\text { Valentina Visconti } \\
\text { Ludwig von Orléans } \\
\text { Johann von Berry } \\
\text { Philipp der Kühne } \\
\text { Limburg, Gebrüder }\end{array}$ & $\begin{array}{l}\text { Karl VI. } \\
\text { Johann Ohnefurcht } \\
\text { Karl I. von Bourbon } \\
\text { Guy de La Rochefoucauld } \\
\text { Johann von Berry }\end{array}$ & $\begin{array}{l}150 \text { fr. } \\
100 \text { fr. } \\
100 \text { l.t. } \\
66 \text { fr. } 5 \text { s.t. } \\
2 \text { l. } 10 \text { s. }\end{array}$ \\
\hline
\end{tabular}

Tab. 21: Zeitgleich-reziprok bestimmte Schenkbeziehungen nach Wertzuweisungen

Wieder ist die Spitzengruppe burgundisch dominiert, nun jedoch mit dem König in einer eindeutigen Führungsposition in der dreimaligen Paarung mit seinem ersten Pair. Kennzeichen der nach Werten bemessenen Bindung scheint hier jedoch ein überwiegend familiäres Moment zu sein. Eindeutig ist aber das Fehlen der Königin, die erst auf dem 19. Rang erscheint. Schließlich erscheint ein diplomatischer reziproker Gabentausch mit hohem Wert, der aber wahrscheinlich kaum Folgen gehabt hatte, ist doch der Partner Philipps, Papst Clemes VII., noch im selben Jahr gestorben. Deutlich zu erkennen ist auch die zumindest in den tabellarisch notierten Werten aufscheinende Kostbarkeit der Gaben. Im Verbund mit der Beobachtung, daß zwar die Spitzenränge mit Blick auf auf den jeweiligen Zusammenhang von Gabe und Gegengabe in ihrer Besetzung eine tendenziell familiäre Orientierung aufweisen, die meisten Paarungen jedoch nicht familiären Nahverhältnissen entsprechen, scheint gegenseitige Reziprozität des Gabentausches auch unter dem Aspekt Gewohnheit der noch deutlicher profilierte zentrale soziale Ort der höfischen Gabe gewesen zu sein. Es muß ein besonderer Ausweis der Ehrzuweisung gewesen sein, in diesen Kreis aufgenommen worden zu sein, wie es den La Trémoilles widerfahren ist. 